\title{
Experimental challenges for the Re/Os clock
}

\author{
Marita Mosconi' ${ }^{* 1}$ M. Heil ${ }^{1}$, F. Käppeler ${ }^{1}$, R. Plag ${ }^{1}$, A. Mengoni ${ }^{3,17}$, K. Fujii ${ }^{4}$, R. Gallino ${ }^{5}$, G. Aerts ${ }^{6}$, R. \\ Terlizzi $^{7}$, U. Abbondanno ${ }^{4}$, H. Álvarez-Pol ${ }^{12}$, F. Alvarez-Velarde ${ }^{6}$, S. Andriamonje ${ }^{6}$, J. Andrzejewski ${ }^{19}$, \\ P. Assimakopoulos ${ }^{8}$, L. Audouin ${ }^{6}$, G. Badurek ${ }^{9}$, P. Baumann ${ }^{10}$, F. Bečváŕr ${ }^{11}$, E. Berthoumieux ${ }^{6}$, \\ S. Bisterzo ${ }^{5,1}$, F. Calviño ${ }^{13}$, D. Cano-Ott ${ }^{14}$, C. Carrapiço ${ }^{16}$, R. Capote $^{3,15}$, P. Cennini ${ }^{17}$, V. Chepel ${ }^{18}$, \\ E. Chiaveri ${ }^{17}$, N. Colonna ${ }^{7}$, G. Cortes ${ }^{13}$, A. Couture ${ }^{20}$, J. Cox $^{20}$, M. Dahlfors ${ }^{17}$, S. David ${ }^{21}$, I. Dillman ${ }^{1}$, \\ R. Dolfini ${ }^{22}$, C. Domingo Pardo ${ }^{40}$ W. Dridi ${ }^{14}$, I. Duran ${ }^{12}$, C. Eleftheriadis ${ }^{23}$, M. Embid-Segura ${ }^{14}$, \\ L. Ferrant $^{21}$, A. Ferrari ${ }^{17}$, R. Ferreira-Marques ${ }^{18}$, L. Fitzpatrick ${ }^{17}$, H. Frais-Koelbl ${ }^{6}$, W. Furman ${ }^{24}$, \\ I. Goncalves ${ }^{18}$, E. Gonzalez-Romero ${ }^{14}$, A. Goverdovski ${ }^{25}$, F. Gramegna ${ }^{26}$, E. Griesmayer ${ }^{6}$, \\ C. Guerrero ${ }^{14}$, F. Gunsing ${ }^{6}$, B. Haas ${ }^{27}$, R. Haight ${ }^{28}$, A. Herrera-Martinez ${ }^{17}$, M. Igashira ${ }^{29}$, S. Isaev ${ }^{21}$, \\ E. Jericha ${ }^{9}$, Y. Kadi ${ }^{17}$, D. Karamanis ${ }^{8}$, D. Karadimos ${ }^{8}$, M. Kerveno, ${ }^{10}$, V. Ketlerov ${ }^{25,17}$, P. Koehler ${ }^{30}$, \\ V. Konovalov ${ }^{24,17}$, E. Kossionides ${ }^{31}$, M. Krtička ${ }^{11}$, C. Lamboudis ${ }^{8}$, H. Leeb ${ }^{9}$, A. Lindote ${ }^{18}$, I. Lopes ${ }^{18}$, \\ M. Lozano ${ }^{15}$, S. Lukic $^{10}$, J. Marganiec ${ }^{19}$, S. Marrone ${ }^{7}$, P. Mastinu ${ }^{26}$, P.M. Milazzo ${ }^{4}$, C. Moreau ${ }^{4}$, \\ F. Neves ${ }^{18}$, H. Oberhummer ${ }^{9}$, M. Oshima ${ }^{32}$, S. O'Brien ${ }^{20}$, J. Pancin ${ }^{6}$, C. Papachristodoulou ${ }^{8}$, \\ C. Papadopoulos ${ }^{33}$, C. Paradela ${ }^{12}$, N. Patronis ${ }^{8}$, A. Pavlik ${ }^{34}$, P. Pavlopoulos ${ }^{35}$, L. Perrot ${ }^{6}$, \\ A. Plompen ${ }^{36}$, A. Plukis ${ }^{6}$, A. Poch ${ }^{13}$, C. Pretel ${ }^{13}$, J. Quesada ${ }^{15}$, T. Rauscher ${ }^{37}$, R. Reifarth ${ }^{28}$, \\ M. Rosetti ${ }^{38}$, C. Rubbia ${ }^{22}$, G. Rudolf ${ }^{10}$, P. Rullhusen ${ }^{36}$, J. Salgado ${ }^{16}$, L. Sarchiapone ${ }^{17}$, I. Savvidis ${ }^{23}$, \\ C. Stephan $^{21}$, G. Tagliente ${ }^{7}$, J.L. Tain ${ }^{40}$, L. Tassan-Got ${ }^{21}$, L. Tavora ${ }^{16}$, G. Vannini $^{39}$, P. Vaz ${ }^{16}$, \\ A. Ventura ${ }^{38}$, D. Villamarin ${ }^{6}$, M. C. Vincente ${ }^{6}$, V. Vlachoudis ${ }^{17}$, R. Vlastou ${ }^{33}$, F. Voss ${ }^{1}$, S. Walter ${ }^{1}$, \\ H. Wendler ${ }^{17}$, M. Wiescher ${ }^{20}$, K. Wisshak $^{1}$
}

\section{The n_TOF Collaboration}

\footnotetext{
${ }^{1}$ Forschungszentrum Karlsruhe GmbH (FZK), Institut für Kernphysik, Germany; ${ }^{3}$ International Atomic Energy Agency, NAPC-Nuclear Data Section, Vienna, Austria; ${ }^{4}$ Istituto Nazionale di Fisica Nucleare, Trieste, Italy; ${ }^{5}$ Dipartimento di Fisica Generale, Università di Torino, Italy; ${ }^{6}$ CEA/Saclay - DSM, Gif-sur-Yvette, France; ${ }^{7}$ Istituto Nazionale di Fisica Nucleare, Bari, Italy; ${ }^{8}$ University of Ioannina, Greece; ${ }^{9}$ Atominstitut der Österreichischen Universitäten,Technische Universität Wien, Austria; ${ }^{10}$ Centre National de la Recherche Scientifique/IN2P3 - IReS, Strasbourg, France; ${ }^{11}$ Charles University, Prague, Czech Republic; ${ }^{12}$ Universidade de Santiago de Compostela, Spain; ${ }^{13}$ Universitat Politecnica de Catalunya, Barcelona, Spain; ${ }^{14}$ Centro de Investigaciones Energeticas Medioambientales y Technologicas, Madrid, Spain; ${ }^{15}$ Universidad de Sevilla, Spain; ${ }^{16}$ Instituto Tecnológico e Nuclear(ITN), Lisbon, Portugal; ${ }^{17}$ CERN, Geneva, Switzerland; ${ }^{18}$ LIP - Coimbra \& Departamento de Fisica da Universidade de Coimbra, Portugal; ${ }^{19}$ University of Lodz, Lodz, Poland; ${ }^{20}$ University of Notre Dame, Notre Dame, USA; ${ }^{21}$ Centre National de la Recherche Scientifique/IN2P3 IPN, Orsay, France; ${ }^{22}$ Università degli Studi Pavia, Pavia, Italy; ${ }^{23}$ Aristotle University of Thessaloniki, Greece; ${ }^{24}$ Joint Institute for Nuclear Research, Frank Laboratory of Neutron Physics, Dubna, Russia; ${ }^{25}$ Institute of Physics and Power Engineering, Kaluga region, Obninsk, Russia; ${ }^{26}$ Istituto Nazionale di Fisica Nucleare(INFN), Laboratori Nazionali di Legnaro, Italy; ${ }^{27}$ Centre National de la Recherche Scientifique/IN2P3 - CENBG, Bordeaux, France; ${ }^{28}$ Los Alamos National Laboratory, New Mexico, USA; ${ }^{29}$ Tokyo Institute of Technology, Tokyo, Japan; ${ }^{30}$ Oak Ridge National Laboratory, Physics Division, Oak Ridge, USA; ${ }^{31}$ NCSR, Athens, Greece; ${ }^{32}$ Japan Atomic Energy Research Institute, Tokai-mura, Japan; ${ }^{33}$ National Technical University of Athens, Greece; ${ }^{34}$ Institut für Isotopenforschung und Kernphysik, Universität Wien, Austria; ${ }^{35}$ Pôle Universitaire Léonard de Vinci, Paris La Défense, France;

${ }^{36}$ CEC-JRC-IRMM, Geel, Belgium; ${ }^{37}$ Department of Physics and Astronomy - University of Basel, Basel, Switzerland;

${ }^{38}$ ENEA, Bologna, Italy; ${ }^{39}$ Dipartimento di Fisica, Università di Bologna, and Sezione INFN di Bologna, Italy;

${ }^{40}$ Instituto de Física Corpuscular, CSIC-Universidad de Valencia, Spain

E-mail: marita.mosconi@ik.fzk.de
} 
The stellar neutron capture cross sections of ${ }^{186} \mathrm{Os}$ and ${ }^{187} \mathrm{Os}$ are fundamental for the Re/Os cosmo-chronometer since they are defining the $s$-process abundance of ${ }^{187} \mathrm{Os}$. Subtraction of the $s$-component from the solar ${ }^{187} \mathrm{Os}$ abundance yields the radiogenic contribution to ${ }^{187} \mathrm{Os}$ due to $\beta$-decays of ${ }^{187} \operatorname{Re}\left(\mathrm{t}_{1 / 2}=42.3 \mathrm{~Gy}\right)$ originating from $r$-process nucleosynthesis. The laboratory cross section of ${ }^{187}$ Os requires a significant correction for the effect of the low-lying excited state at $9.75 \mathrm{keV}$, which is strongly populated under stellar conditions. This reliability of the theoretical correction can be greatly improved by an experimental determination of the cross section for inelastic scattering to the $9.75 \mathrm{keV}$ state. In this work, high resolution time-of-flight measurements of $(n, \gamma)$ cross sections of ${ }^{186,187,188} \mathrm{Os}$ from $1 \mathrm{eV}$ to $1 \mathrm{MeV}$ performed at the CERN n_TOF facility are reported. The inferred stellar cross sections differ from previously recommended values. In addition, the inelastic scattering cross section has been measured at $30 \mathrm{keV}$ neutron energy via time-of-flight at the Karlsruhe 3.7 MV Van de Graaff. The implications of these results for the Re/Os clock are briefly discussed.

International Symposium on Nuclear Astrophysics - Nuclei in the Cosmos - IX 25-30 June 2006

CERN

*Speaker. 


\section{Introduction}

The Re/Os cosmochronometer (proposed by Clayton [1]) represents a nuclear dating technique independent of astronomical observations and - apart from a realistic model of galactic chemical evolution - firmly based on nuclear reaction and decay properties. The temperature dependence of the ${ }^{187} \mathrm{Re}$ half-life has been determined by a measurement of the half-life of fully stripped ${ }^{187} \mathrm{Re}$ atoms [2], and the resulting consequence for the reliability of the clock was found to be relatively small [3]. Hence, only the Maxwellian averaged cross sections (MACS) for neutron capture on ${ }^{186}$ Os and ${ }^{187}$ Os under stellar conditions need to be experimentally investigated. In turn, the required calculation of the stellar enhancement factor of the ${ }^{187}$ Os cross section needs the support of a measurement of the inelastic scattering cross section.

Previous measurements of the neutron capture cross sections of the stable Os isotopes ${ }^{186-190} \mathrm{Os}$ and of ${ }^{192} \mathrm{Os}[4,5]$ suffered from the fact that the astrophysically relevant energy range was not fully covered. This holds in particular for the low energy region, which is important for most of the $s$ process neutron exposure provided by the ${ }^{13} \mathrm{C}(\alpha, n){ }^{16} \mathrm{O}$ reaction. Among the two cross section sets, the MACS of ${ }^{186}$ Os exhibits some discrepancies, especially at energies close to the first excited state of ${ }^{186} \mathrm{Os}$ at $130 \mathrm{keV}$, where a Hauser-Feshbach calculation is suggesting a pronounced step in the cross section (Fig. 1). Consequently, improved capture cross section measurements are called for to solve existing discrepancies and to extend the energy range to lower and higher energies for obtaining a more reliable energy dependence of the $(n, \gamma)$ cross sections.

The available measurements of the inelastic scattering cross section of ${ }^{187}$ Os $[6,7,8]$ also exhibit large uncertainties that are limiting the reliability of the calculated stellar enhancement factor. Therefore, an accurate independent measurement of the $\left(n, n^{\prime}\right)$ cross section of ${ }^{187} \mathrm{Os}$ is another experimental challenge for an improved assessment of the Re/Os cosmochronometer.

\section{Neutron capture measurements at n_TOF}

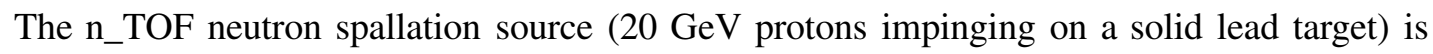
producing neutrons in the energy range from thermal to above $1 \mathrm{GeV}$, with neutron capture measurements commonly performed in the energy range from $1 \mathrm{eV}$ to $1 \mathrm{MeV}$. Due to the flight path of $187.5 \mathrm{~m}$ and the short proton burst width of $6 \mathrm{~ns}$, the time of flight (TOF) method yields an excellent neutron energy resolution of $3 \times 10^{-4}$ and $4 \times 10^{-3}$ at $1 \mathrm{eV}$ and $1 \mathrm{MeV}$, respectively [9]. In the experimental area the isolethargic neutron flux varies smoothly with neutron energy, and the total fluence ranges from $4.5 \times 10^{4}$ to $2 \times 10^{5}$ neutrons per proton bunch between $1 \mathrm{eV}$ and $1 \mathrm{MeV}$ [9].

Each of the Os samples consisted of $2 \mathrm{~g}$ of enriched metal powder on loan from Oak Ridge National Laboratory. In fact, this was the same material as used in previous experiments $[5,4]$. The ${ }^{186} \mathrm{Os}$ and ${ }^{187} \mathrm{Os}$ samples were enriched to $79 \%$ and $70 \%$ with a ${ }^{188}$ Os content of $5 \%$ and $\sim 13 \%$, respectively. The $(n, \gamma)$ cross section of ${ }^{188}$ Os was measured as well using a $95 \%$ enriched sample in order to account for the main isotopic correction. The impurities due to ${ }^{189} \mathrm{Os},{ }^{190} \mathrm{Os}$, and ${ }^{192}$ Os were all around $5 \%$. The sample material was encapsulated in thin aluminium cans, 15 $\mathrm{mm}$ in diameter. 

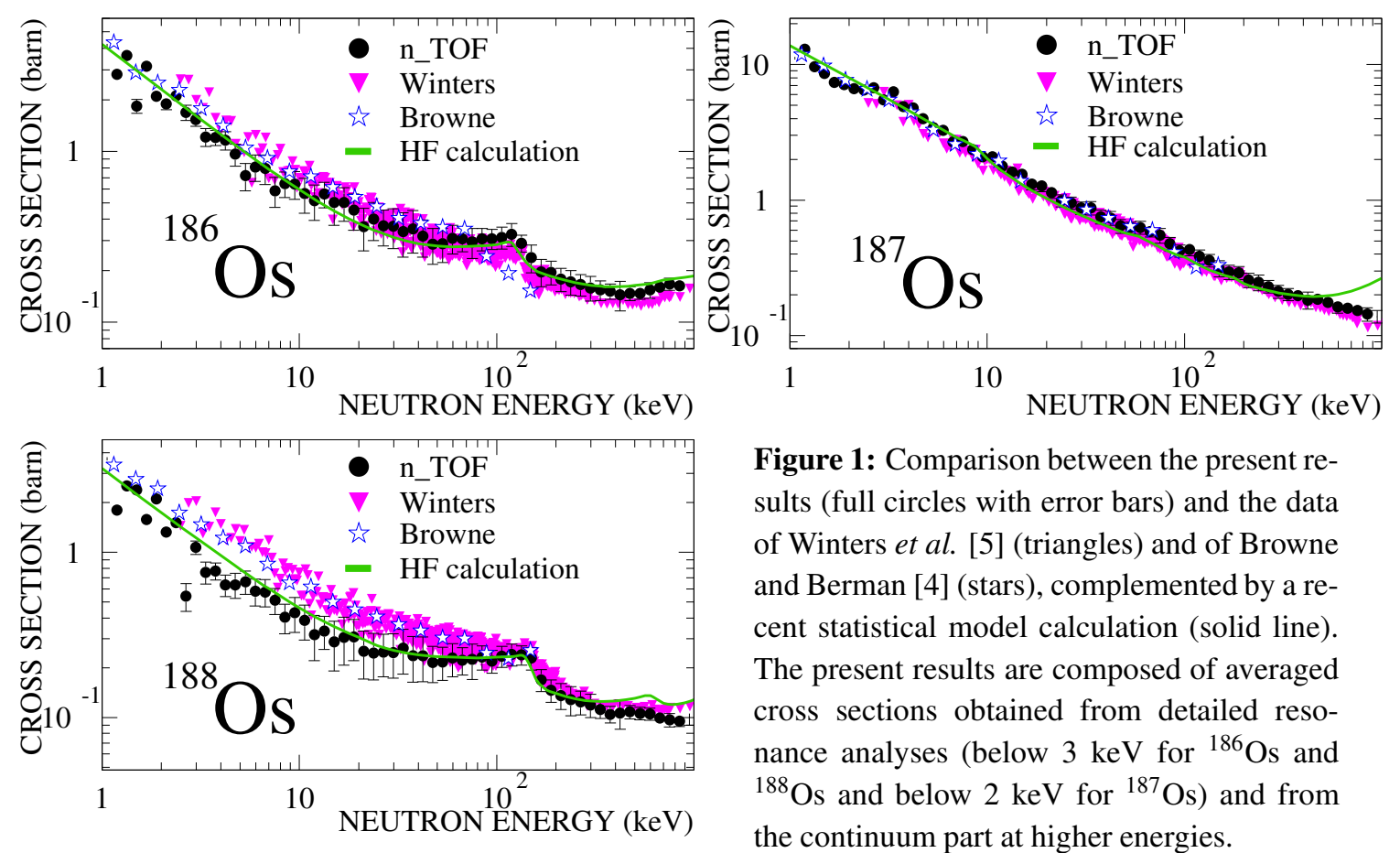

Figure 1: Comparison between the present results (full circles with error bars) and the data of Winters et al. [5] (triangles) and of Browne and Berman [4] (stars), complemented by a recent statistical model calculation (solid line). The present results are composed of averaged cross sections obtained from detailed resonance analyses (below $3 \mathrm{keV}$ for ${ }^{186} \mathrm{Os}$ and ${ }^{188}$ Os and below $2 \mathrm{keV}$ for ${ }^{187} \mathrm{Os}$ ) and from the continuum part at higher energies.

The $\gamma$-ray cascades from capture events have been detected with two $\mathrm{C}_{6} \mathrm{D}_{6}$ scintillators $(12 \mathrm{~cm}$ in diameter and each with a volume of $\approx 11$ ), which were placed perpendicular to the neutron beam. A neutron monitor consisting of a thin ${ }^{6} \mathrm{Li}$ foil was used for flux normalization, which was surrounded by a set of four Si detectors mounted outside the neutron beam for recording the products of the ${ }^{6} \mathrm{Li}(n, \alpha)^{3} \mathrm{H}$ reaction. The $\gamma$-detectors were designed to minimize the neutron sensitivity [10]. The signals from the $\mathrm{C}_{6} \mathrm{D}_{6}$ scintillators were recorded with flash amplitude-todigital converters (FADC) for off-line application of the pulse height weighting technique (PHWT) [11]. In this way, the detection efficiency was obtained to be independent of the multiplicity of the prompt capture $\gamma$-ray cascade. The Os samples were complemented by a set of samples that served for evaluation of the background and normalization. These were a carbon sample for the effect of scattered neutrons, a lead sample for the scattering of in-beam $\gamma$-rays, a gold sample for neutron flux normalization, and an empty position for obtaining the ambient background component.

All samples were mounted on the ladder of a remotely controlled sample changer. Normalization to equal neutron flux was achieved by means of the Si neutron monitor and by calibration runs with the gold sample. The cross sections were normalized to the gold reference cross section using the accurate experimental average value at $25 \mathrm{keV}$ [12]. The background components in the spectra of the Os samples and of the gold sample have been treated individually. In particular, the weighting functions were determined by a complete simulation of the experimental setup, including the effect of the sample itself. The evaluated background has been validated by comparison with spectra obtained with black neutron filters in the beam.

The analysis of the data and the calculation of the cross section were treated in the resolved resonance region and in the continuum part in different ways. The resolved resonance region has been analyzed by the R-matrix code SAMMY. Individual resonance parameters were considered 
up to $3 \mathrm{keV}$ neutron energy for ${ }^{186} \mathrm{Os}$ and ${ }^{188} \mathrm{Os}$ and up to $2 \mathrm{keV}$ for ${ }^{187} \mathrm{Os}$.

The cross section in the continuum has been determined by subtracting the properly normalized background and by applying the corrections for self-absorption and multiple scattering obtained with the SESH code [13] as well as the respective isotopic corrections. The background obtained by the SAMMY analysis was found in excellent agreement with the result of the direct measurement and provided an independent confirmation of the background subtraction method used.

The final cross sections plotted in Fig. 1 were calculated by combining the average data from the resonance analysis and from the continuum region. The comparison in Fig. 1 shows good agreement with previous data for ${ }^{186} \mathrm{Os}$ and ${ }^{187} \mathrm{Os}$, whereas significant differences with respect to previous measurements are found for ${ }^{188} \mathrm{Os}$. Up to $400 \mathrm{keV}$ the present results are well described by the Hauser-Feshbach statistical model calculations.
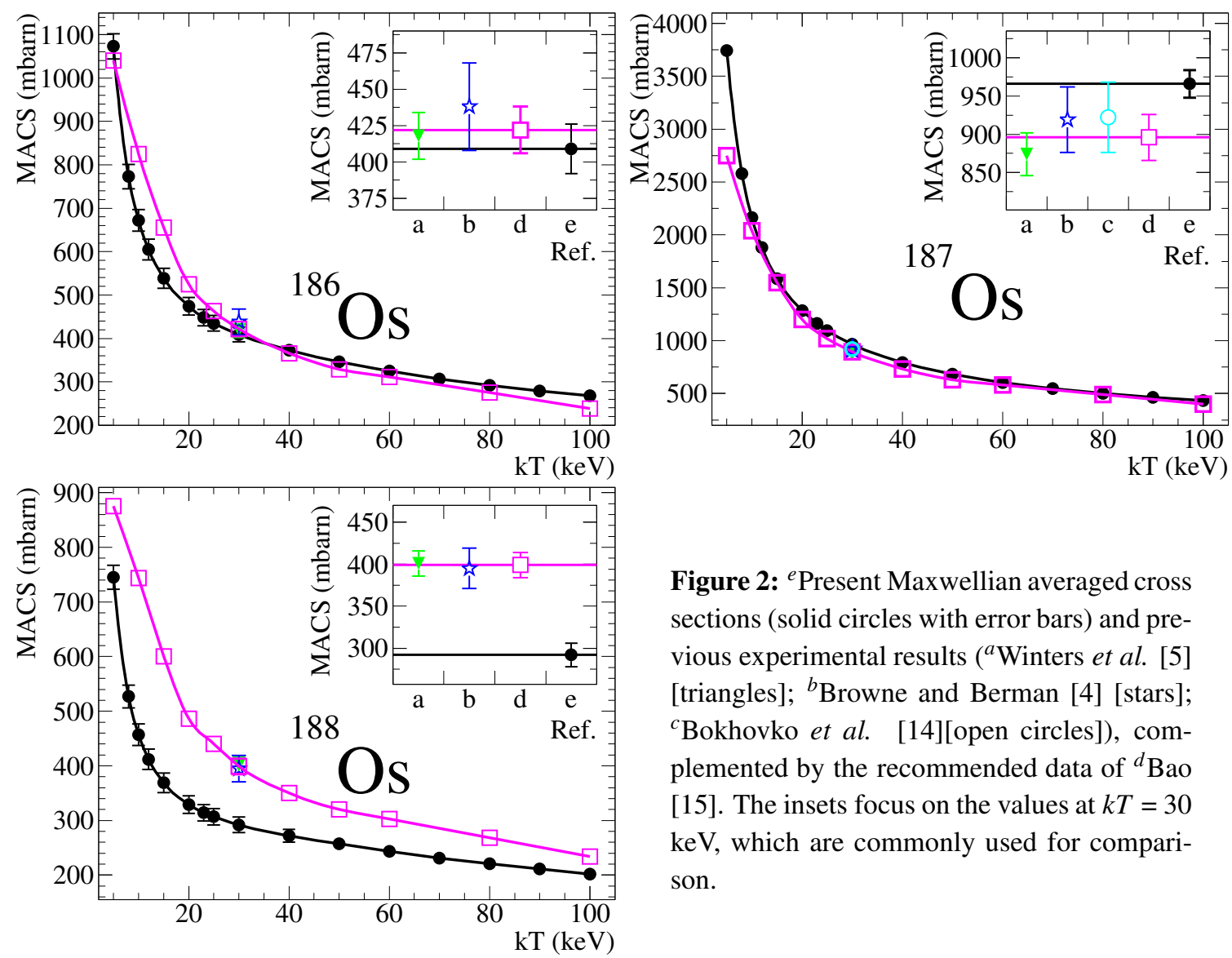

Figure 2: ${ }^{e}$ Present Maxwellian averaged cross sections (solid circles with error bars) and previous experimental results $\left({ }^{a}\right.$ Winters et al. [5] [triangles]; ${ }^{b}$ Browne and Berman [4] [stars]; ${ }^{c}$ Bokhovko et al. [14][open circles]), complemented by the recommended data of ${ }^{d}$ Bao [15]. The insets focus on the values at $k T=30$ $\mathrm{keV}$, which are commonly used for comparison.

The MACSs have been evaluated for thermal energies from $5 \mathrm{keV}$ to $100 \mathrm{keV}$ (Fig. 2). At $k T$ $=30 \mathrm{keV}$, the present results for ${ }^{188} \mathrm{Os}$ is $27 \%$ lower than the recommended value [15], whereas that for ${ }^{186} \mathrm{Os}$ is in agreement with all previous measurements and with the recommendation of Ref. [15]. For ${ }^{187}$ Os the present result is in agreement with the data of Browne and Berman [4] and of Bokhovko et al. [14], but 7\% higher than that of Ref. [15].

During the $s$ process reaction flow equilibrium has been locally established from the mass region of the rare earth elements up to $A=190$ due to the comparably large MACSs of the involved 
isotopes. This implies that the local approximation $N_{s} \times\langle\sigma\rangle=$ constant holds to a very good approximation. Comparison of the $N_{s} \times\langle\sigma\rangle$ products of the two $s$-only isotopes ${ }^{186} \mathrm{Os}$ and ${ }^{187} \mathrm{Os}$ can, therefore, be used for deriving the excess ${ }^{187} \mathrm{Os}$ from the measured ratio of the stellar MACS of ${ }^{187} \mathrm{Os}$ and ${ }^{186} \mathrm{Os}$. This excess corresponds to the radiogenic component due to the decay of the long-lived ${ }^{187} \mathrm{Re}$.

Based on the measured cross sections, the MACS ratio is $186 / 187=0.42 \pm 0.02$. Under the assumption of the coarse Fowler model [16] for galactic chemical evolution (GCE) and neglecting the time prior to the formation of our Galaxy, the age of the Universe is estimated to be around 14.5 Gyr. By including the stellar enhancement factor for the MACS of ${ }^{187}$ Os from Ref. [15], this ratio reduces to $0.36 \pm 0.02$, yielding a slightly higher age of $16 \pm 2 \mathrm{Gyr}$, somewhat higher than the U/Th age of $14.5 \pm{ }_{2.2}^{2.8}$ Gyr [17] and than the results based on cosmological evidence [18]. This very preliminary result has yet to be verified by means of an improved evaluation of the stellar enhancement factor and by using a more realistic galactic evolution model.

\section{Inelastic scattering experiment at FZK}

The neutron inelastic scattering cross section of ${ }^{187}$ Os has been measured at the $3.7 \mathrm{MV}$ Van de Graaff of Forschungszentrum Karlsruhe using the ${ }^{7} \mathrm{Li}(p, n)^{7} \mathrm{Be}$ reaction for obtaining a "monoenergetic" neutron beam of $30 \mathrm{keV}$. The energy of the pulsed proton beam $(\Delta \mathrm{t}=10 \mathrm{~ns})$ was adjusted right at the $(p, n)$ threshold to produce kinematically collimated neutrons, which are emitted in a narrow cone with an opening angle of not more than $13 \mathrm{deg}$ and an energy spread of less than 10 $\mathrm{keV}$. In this way, neutron collimators could be avoided because the detectors for scattered neutrons could be placed outside the main neutron beam. The $\gamma$-ray flash from the impact of the proton pulse on the ${ }^{7} \mathrm{Li}$ target was reduced by a small lead shield around the target.
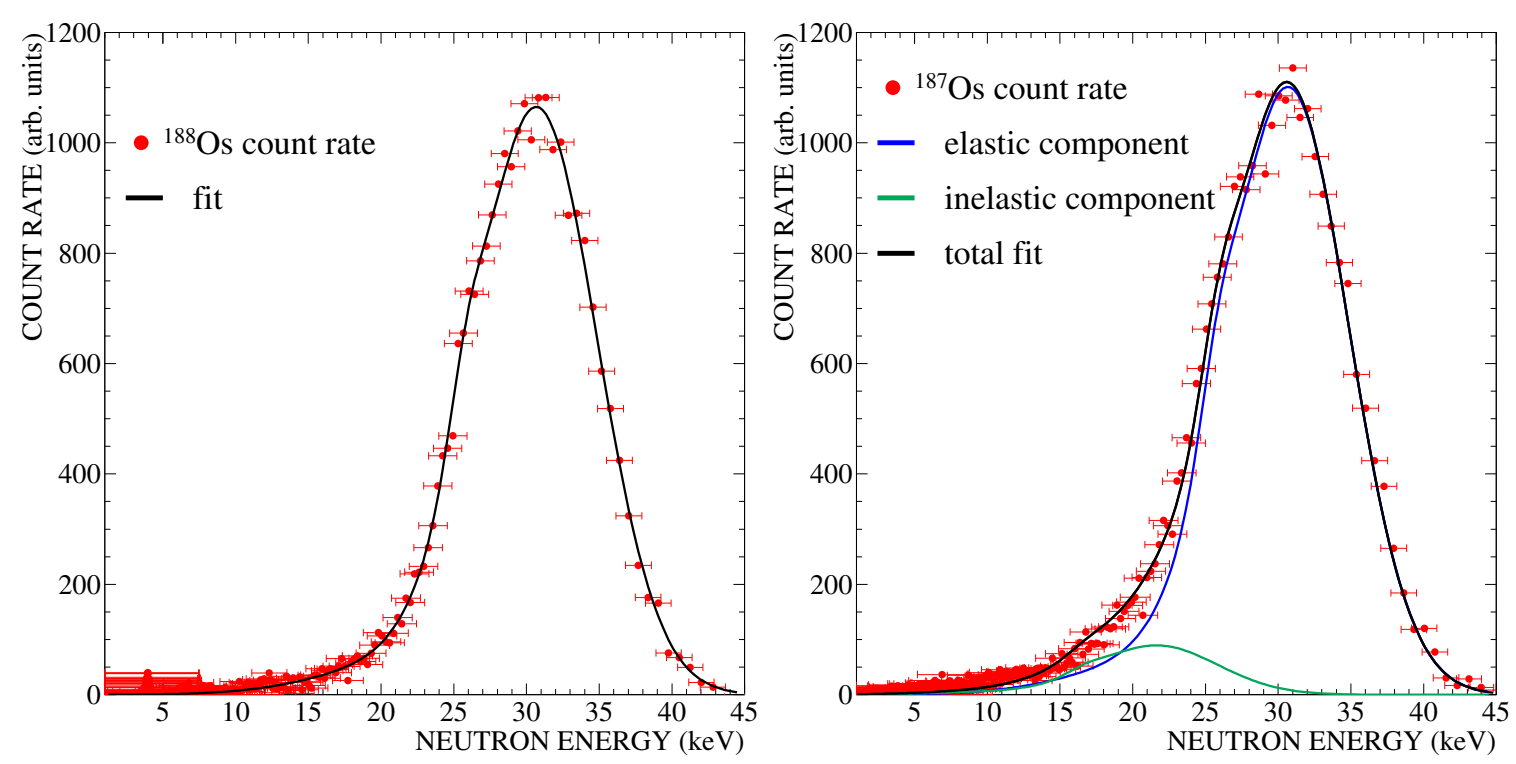

Figure 3: Spectra of scattered neutrons from ${ }^{188} \mathrm{Os}$ (left) and ${ }^{187} \mathrm{Os}$ (right) corresponding to a neutron beam of $9.5 \mathrm{keV}$ FWHM. The signature of the inelastic component is clearly visible in the spectrum of ${ }^{187} \mathrm{Os}$, suggesting that the inelastic scattering cross section is more than 10 times smaller than the elastic part. 
The measurement have been performed with the same ${ }^{187} \mathrm{Os}$ and ${ }^{188} \mathrm{Os}$ samples as used in the neutron capture experiment in order to avoid losses of the expensive material. The samples were mounted $4 \mathrm{~cm}$ in front of the Li target under an angle of $45 \mathrm{deg}$ with respect to the proton beam in order to minimize self shielding effects. Scattered neutrons were detected by three ${ }^{6} \mathrm{Li}$-glass scintillators $3 \mathrm{~mm}$ in thickness and $12 \mathrm{~cm}$ in diameter, two at $90 \mathrm{deg}$ and one at $75 \mathrm{deg}$ relative to the direction of the primary beam, all placed at a distance of $26 \mathrm{~cm}$ from the scattering sample. An additional $3 \mathrm{~mm}$ thick ${ }^{6} \mathrm{Li}$-glass scintillator $38 \mathrm{~mm}$ in diameter, which was mounted in forward direction at $111 \mathrm{~cm}$ from the ${ }^{7} \mathrm{Li}$ target, served as a monitor for the primary neutron flux. The data acquisition system consisted of a set of four FADCs, which had the advantage of a detailed off-line analysis of the recorded signals. In this way, the signal/background ratio could be improved by pulse shape discrimination between neutrons and $\gamma$-rays.

During the measurements the proton energy was tuned such that the FWHM of the neutron energy distribution was below $10 \mathrm{keV}$. Due to the normal charge fluctuation of the Van de Graaff, the width of the distribution varied between 7 and $10 \mathrm{keV}$ in cycles of several hours.

The separation of the elastic and inelastic scattering component was obtained by measuring the neutron energies via TOF and by using the elastic component from the scattering spectrum of ${ }^{188}$ Os. The inelastic part can then be extracted by a fit assuming that the inelastic component is represented by the same distribution shifted in energy by the $9.75 \mathrm{keV}$ excitation energy of the first excited state of ${ }^{187} \mathrm{Os}$. In this way, the fit contains only two free parameters, namely the amplitudes of the elastic and inelastic distributions. This analysis is in progress; a preliminary result is shown in Fig. 3.

\section{Conclusions and remarks}

The challenges related to the neutron capture cross section measurements have been met: the $n_{-}$TOF cross sections are available in the entire range of astrophysical interest with typical uncertainties of 5\%. The data exhibit clear signatures for the competition of the inelastic channel, which had previously not been found for ${ }^{186} \mathrm{Os}$. A final statement concerning the Re/Os clock must await completion of the analysis of the inelastic scattering cross section. Presently, the data seem to indicate an older age of the universe than obtained with other methods, but this may well be due to the very crude GCE model used so far.

\section{Acknowledgments}

This work is supported by National Institutions of the related n_TOF participants and partially by EC under contract FIKW-CT-2000-00107.

\section{References}

[1] Clayton, D. D., Ap. J., 139, 637 - 663 (1964).

[2] Bosch, F. et al., Phys. Rev. Lett., 77, 5190 - 5193 (1996).

[3] Takahashi, K., The ${ }^{187}$ Re - ${ }^{187}$ Os cosmochronometry - the latest developments, in Tours Symposium on Nuclear Physics III, edited by M. Arnould, M. Lewitowicz, Y. Oganessian, M. Ohta,

H. Utsunomiya, and T. Wada, (AIP, New York, 1998), pp. 616- 625. 
[4] Browne, J. C. and Berman, B. L., Phys. Rev. C, 23, 1434 (1981).

[5] Winters, R. R., Macklin, R. L., and Halperin, J., Ap. J., 233, 411-417 (1979).

[6] Hershberger, R., Macklin, R., Balakrishnan, M., Hill, N., and McEllistrem, M., Phys. Rev. C, 28, 2249 -2258 (1983).

[7] Macklin, R. L., Winters, R. R., Hill, N. W., and Harvey, J. A., Ap. J., 274, 408 (1983).

[8] http://www.nndc.bnl.gov/exfor3/exforO0.htm.

[9] The n_TOF collaboration, CERN n_TOF facility: Performance Report, Report CERN/INTC-O-011, CERN, Switzerland (2002).

[10] Plag, R., Heil, M., Käppeler, Pavlopoulos, P., Reifarth, R., and Wisshak, K., Nucl. Instr. Meth. A, 496, $425-436$ (2003).

[11] Aerts, G., Berthoumiex, E., Gunsing, F., and Perrot, L., Weighting functions for the neutron capture measurements performed at n_TOF-CERN in 2002-2003, Report DAPNIA-04-106, CEA/Saclay, France (2004).

[12] Ratynski, W. and Käppeler, F., Phys. Rev. C, 37, 595 - 604 (1988).

[13] Fröhner, F. H., General Atomic Report, GA-8380 (1968).

[14] Bokhovko, M.V., Kononov, V.N., Poletaev, E.D., Rabotnov, N.S., and Timokhov, V.M., Average fast neutron radiative capture cross-sections for fission products and for isotopes of rare earth elements, in Nuclear Data for Science and Technology, edited by S.M. Qaim, (Springer, Berlin, 1992) p. 62.

[15] Bao, Z. Y., Beer, H., Käppeler, F., Voss, F., and Wisshak, K., At. Data Nucl. Data Tables, 76, 70 - 154 (2000).

[16] Rolfs, C. E. and Rodney, W. S, Cauldrons in the Cosmos, (The University of Chicago Press, Chicago (1996).

[17] Dauphas, N., Nature 435, 1203 (2005).

[18] Bennett C. L. et al., Ap. J., 148, 1 (2003). 